\title{
ON A RELATION BETWEEN ABSOLUTE ABEL AND ABSOLUTE RIESZ SUMMABILITY
}

\section{J. S. RATTI}

1.1. Let $\sum a_{n}$ be an infinite series, and let $\left\{\lambda_{n}\right\}$ be an arbitrary sequence of positive numbers tending to infinity with $n$ such that $1 \leqq \lambda_{1}<\lambda_{2}<\cdots$. We write

$$
\begin{aligned}
& A_{\lambda}^{k}(x)=\sum_{\lambda_{n}<x}\left(x-\lambda_{n}\right)^{k} a_{n}=\int_{1}^{x}(x-t)^{k} d A_{\lambda}(t), \\
& A_{\lambda}^{0}(x)=A_{\lambda}(x)=\sum_{\lambda_{n}<x} a_{n}, \\
& A_{\lambda}^{k}(x)=0 \quad \text { for } x \leqq 1 \text { and } k>-1 .
\end{aligned}
$$

We alșo write

$$
B_{\lambda}^{k}(x)=\sum_{\lambda_{n}<x}\left(x-\lambda_{n}\right)^{k} \lambda_{n} a_{n}
$$

The given series $\sum a_{n}$ is said to be summable $(R, \lambda, k)$ to the sum $s$ if $\lim x^{-k} A_{\lambda}^{k}(x)=s$ as $x \rightarrow \infty$; the series is said to be absolutely Riesz summable with index $m$, or simply $|R, \lambda, k|_{m}$ summable [6] if

$$
\int_{1}^{\infty} x^{m-1}\left|\frac{d}{d x} x^{-k} A_{\lambda}^{k}(x)\right|^{m} d x<\infty,
$$

where $k>0, m \geqq 1$ and $k>1-1 / m$.

We say that the given series is summable $|R, \lambda, k, \gamma|_{m}[6]$ if

$$
\int_{1}^{\infty} x^{m \gamma+m-1}\left|\frac{d}{d x} x^{-k} A^{k}(x)\right|^{m} d x<\infty,
$$

where $k>1-1 / m, k>\gamma-1$ and $\gamma$ is a real number. It is evident that $|R, \lambda, k, 0|_{m}$ summability is the same as $|R, \lambda, k|_{m}$ summability and $|R, \lambda, k|_{1}$ summability is the usual absolute Riesz summability denoted by $|R, \lambda, k|$.

The series $\sum a_{n}$ is said to be summable $|A, \lambda|_{m}, m \geqq 1$, if the series $f(x)=\sum a_{n} \exp \left[-\lambda_{n} x\right]$ converges for $x>0$ and

$$
\int_{0}^{\infty}\left(1-e^{-x}\right)^{m-1}\left|f^{\prime}(x)\right|^{m} d x<\infty
$$

[9, Theorem 2].

Received by the editors March 6, 1968. 
Summability $|A, \lambda|_{m}$ can be further generalized by introducing a real parameter $\gamma$ as follows:

For $m \geqq 1$ and $\gamma$ a real number, the series $\sum a_{n}$ is said to be summable $|A, \lambda, \gamma|_{m}$ if

(i) $f(x)=\sum a_{n} \exp \left[-\lambda_{n} x\right]$ converges for $x>0$, and

(ii) $\int_{0}^{\infty}\left(1-e^{-x}\right)^{m-m \gamma-1}\left|f^{\prime}(x)\right| m d x<\infty$.

It is obvious that summability $|A, \lambda, 0|_{m}$ is the same as summability $|A, \lambda|_{m}$ and $|A, \lambda|_{1}$ summability is the same as generalized absolute Abel summability denoted by $|A, \lambda| .(|A, \lambda|$ summability is defined implicitly in [10].)

1.2. In [8] we proved the following theorem.

TheOREM A. If (i) $\sum a_{n}$ is summable $|R, \lambda, k|_{m}$ and (ii) the series $\sum a_{n} \exp \left[-\lambda_{n} x\right]$ converges for all $x>0$ to the sum $f(x)$, then (iii) $\sum a_{n}$ is summable $|A, \lambda|_{m}$.

In the special case $\lambda_{n}=n$, it follows from the results of Borwein [1] and Flett [3] that (i) implies (iii); so that, in this case, (i) alone implies (iii), but this is not true for arbitrary $\lambda_{n}$ (see Remark below).

2.1. We prove the following theorems:

THEOREM 1. If (i) $\sum a_{n}$ is summable $|R, \lambda, k, \gamma|_{m}, m \geqq 1, k>1$ $-1 / m, 0 \leqq \gamma \leqq 1-1 / m$ and (ii) the series $\sum a_{n} \exp \left[-\lambda_{n} x\right]$ converges for all $x>0$ to the sum $f(x)$, then (iii) $\sum a_{n}$ is summable $|A, \lambda, \gamma|_{m}$.

THEOREM 2. For $0<\gamma \leqq 1-1 / n, k>2-1 / m, m>1$, summability $|R, \lambda, k, \gamma|_{m}$ of $\sum a_{n}$ implies its $|A, \lambda, \gamma|_{m}$ summability if and only if $\lim \inf _{n \rightarrow \infty} \exp \left[\lambda_{n} x\right]\left(\lambda_{n+1}-\lambda_{n}\right)>0$ for any $x>0$.

2.2. We require the following lemmas for the proofs of our theorems.

Lemma 1 [5]. If $k>0$, then

$$
\frac{d}{d x}\left(x^{-k} A_{\lambda}^{k}(x)\right)=k x^{-k-1} B_{\lambda}^{k-1}(x) .
$$

Lemma 2 [4, p. 27]. If $k>-1, p>0$, then

$$
A_{\lambda}^{k+p}(x)=M \int_{1}^{x}(x-t)^{p-1} A_{\lambda}^{k}(t) d t .
$$

Throughout this paper $M$ denotes a positive constant, not necessarily the same at every occurrence and $m^{\prime}$ is given by $1 / m+1 / m^{\prime}=1$ for $m>1$. 
LEMмA 3 [7]. Let $\gamma>\mu, m>p \geqq 1$. If $\sum a_{n}$ is summable $|R, \lambda, k, \gamma|_{m}$, then it is also summable $|R, \lambda, k, \mu|_{p}$.

Lemma $4\left[4\right.$, p. 39]. If $\sum a_{n} \exp \left[-\lambda_{n} x\right]$ converges for $x>0$ to the sum $f(x)$, then for $k \geqq 0$,

$$
f(x)=M x^{k+1} \int_{0}^{\infty} A_{\lambda}^{k}(t) e^{-x t} d t .
$$

LEMMA 5. Let $h>0, \gamma \geqq 0, m \geqq 1$. If $\sum a_{n}$ is summable $|R, \lambda, h, \gamma|_{1}$, then it is also summable $|R, \lambda, k, \gamma|_{m}$ for $k>h+1-1 / m, h>\gamma-1$.

Proof of Lemma 5. On account of Lemma 1, we need to show that

$$
I=\int_{1}^{\infty} x^{m \gamma-m k-1}\left|B_{\lambda}^{k-1}(x)\right|^{m} d x<\infty,
$$

given that

$$
J=\int_{1}^{\infty} x^{\gamma-h-1}\left|B_{\lambda}^{h-1}(x)\right|^{m} d x<\infty .
$$

Let $k=h+\delta$, where $\delta>1-1 / m$.

By Lemma 2, we have

$$
B_{\lambda}^{k-1}(x)=M \int_{1}^{x}(x-t)^{\delta-1} B_{\lambda}^{h-1}(t) d t .
$$

Thus for $m>1$

$$
\begin{aligned}
& \left|B_{\lambda}^{k-1}(x)\right| \leqq M \int_{1}^{x}(x-t)^{\delta-1}\left|B_{\lambda}^{h-1}(t)\right| d t \\
& \quad=M \int_{1}^{x}(x-t)^{\delta-1} t^{(h+1-\gamma) / m} t^{\prime(h+1-\gamma) / m}\left|B_{\lambda}^{h-1}(t)\right|^{1 / m}\left|B_{\lambda}^{h-1}(t)\right|^{1 / m} d t .
\end{aligned}
$$

Applying Holder's inequality we get

$$
\begin{aligned}
\left|B_{\lambda}^{k-1}(x)\right|^{m} \leqq & M\left\{\int_{1}^{x}(x-t)^{m(\delta-1)} t^{(h+1-\gamma)(m-1)}\left|B_{\lambda}^{h-1}(t)\right| d t\right\} \\
& \cdot\left\{\int_{1}^{x} t^{\gamma-k-1}\left|B_{\lambda}^{h-1}(t)\right| d t\right\}^{m-1} \\
& \leqq M \int_{1}^{x}(x-t)^{m(\delta-1)} t^{(h+1-\gamma)(m-1)}\left|B_{\lambda}^{h-1}(t)\right| d t
\end{aligned}
$$

since 


$$
\int_{1}^{x} t^{\gamma-h-1}\left|B_{\lambda}^{h-1}(t)\right| d t \leqq J<\infty .
$$

Therefore,

$$
\begin{aligned}
I & \leqq M \int_{1}^{\infty} x^{m \gamma-m h-m \delta-1} d x \int_{1}^{x}(x-t)^{m \delta-m} t^{(h+1-\gamma)(m-1)}\left|B_{\lambda}^{h-1}(t)\right| d t \\
& =M \int_{1}^{\infty} t^{(h+1-\gamma)(m-1)}\left|B_{\lambda}^{h-1}(t)\right| d t \int_{t}^{\infty}(x-t)^{m \delta-m} x^{m \gamma-m h-m \delta-1} d x \\
& =M \int_{1}^{\infty} t^{\gamma-h-1}\left|B_{\lambda}^{h-1}(t)\right| d t=M J<\infty .
\end{aligned}
$$

The case $m=1$ is similar.

LEMMA 6. If $\sum a_{n}$ is summable $|R, \lambda, k, \gamma|_{m}$, then it is also summable $|R, \lambda, h, \gamma|_{m}$ for $k>h$.

The proof of Lemma 6 is similar to that of Lemma 3 in [7] and is therefore omitted.

2.3. Proof of Theorem 1. In virtue of (ii),

$$
f^{\prime}(x)=-M x^{k+1} \int_{0}^{\infty} B_{\lambda}^{k}(t) e^{-x t} d t
$$

Thus

$$
\begin{aligned}
& \int_{0}^{\infty}\left(1-e^{-x}\right)^{m-m \gamma-1}\left|f^{\prime}(x)\right|^{m} d x \\
& \leqq M \int_{0}^{\infty}\left(1-e^{-x}\right)^{m-m \gamma-1} x^{m k+m} d x \\
& \cdot\left\{\int_{0}^{\infty}\left|B_{\lambda}^{k}(t)\right|^{m} e^{-x t} d t\right\}\left\{\int_{0}^{\infty} e^{-x t} d t\right\}^{m-1} \\
& =M \int_{0}^{\infty}\left|B_{\lambda}^{k}(t)\right|^{m} d t \int_{0}^{\infty}\left(\frac{1-e^{-x}}{x}\right)^{m-m \gamma-1} x^{m k+m-m \gamma} e^{-x t} d t \\
& \leqq M \int_{0}^{\infty} x^{m \gamma-m k-m-1}\left|B_{\lambda}^{k}(t)\right|^{m} d t \\
& <\infty \text {, }
\end{aligned}
$$

by Lemma 1 and Lemma 6 with $h=k+1$.

Proof of Theorem 2. Suppose 


$$
\sum a_{n} \text { is summable }|R, \lambda, k, \gamma|_{m}
$$

and

$$
\liminf _{n \rightarrow \infty} \exp \left[\lambda_{n} x\right]\left(\lambda_{n+1}-\lambda_{n}\right)>0 \quad \text { for any } x>0 \text {. }
$$

By Lemma 3, it is clear that $|R, \lambda, k, \gamma|_{m}$ summability of $\sum a_{n}$ implies its $|R, \lambda, k|$ summability, which in turn implies its $(R, \lambda, k)$ summability. If $x_{k}(k \geqq 0)$ denotes the abscissa of $(R, \lambda, k)$ summability of the Dirichlet series

$$
f(x)=\sum a_{n} \exp \left[-\lambda_{n} x\right]
$$

then the assertion that $\sum a_{n}$ is summable $(R, \lambda, k)$ implies $x_{k} \leqq 0$; while the convergence of (3) for all $x>0$ will follow if $x_{0} \leqq 0$. It therefore follows from Theorem 3.52 and Corollary 3.75 of [2] that the hypothesis of Corollary 3.75 is sufficient to ensure the convergence of (3) for all $x>0$ if $\sum a_{n}$ is summable $(R, \lambda, k)$. The hypothesis of Corollary 3.75 of [2], while expressed in a somewhat different form, may easily be seen to be equivalent to condition (2). Thus the sufficiency part of Theorem 2 is given by Theorem 1 .

NECESSITy. Suppose condition (2) on $\left\{\lambda_{n}\right\}$ is not satisfied, then for some $x_{0}>0$,

$$
\liminf _{n \rightarrow \infty} \exp \left[\lambda_{n} x_{0}\right]\left(\lambda_{n+1}-\lambda_{n}\right)=0 .
$$

We can find a sequence $\left\{n_{m}\right\}$ of positive integers such that $n_{m+1}$ $\geqq n_{m}+2$ and such that

$$
\sum_{m=0}^{\infty} \exp \left[\lambda\left(n_{m}\right) x_{0}\right]\left[\lambda\left(n_{m}+1\right)-\lambda\left(n_{m}\right)\right]<\infty,
$$

where we write $\lambda(n)$ for $\lambda_{n}$ whenever $n$ is replaced by an expression which itself involves suffixes.

Define

$$
\begin{array}{rlrl}
a_{n} & =\exp \left[\lambda\left(n_{m}\right) x_{0}\right] & & \left(n=n_{m}\right), \\
& =-\exp \left[\lambda\left(n_{m}\right) x_{0}\right] & & \left(n=n_{m}+1\right), \\
& =0 \quad \text { otherwise; } &
\end{array}
$$

then $f(x)=\sum a_{n} \exp \left[-\lambda_{n} x\right]$ clearly diverges for $x \leqq x_{0}$. Thus $\sum a_{n}$ is not summable $|A, \lambda, \gamma|_{m}$. On the other hand, the series $\sum a_{n}$ is summable $|R, \lambda, k, \gamma|_{m}$. To prove this, on account of Lemma 5 , it is sufficient to prove that $\sum a_{n}$ is summable $|R, \lambda, 1, \gamma|_{1}$. 


$$
\begin{aligned}
& \int_{1}^{\infty} u^{\gamma}\left|\frac{d}{d u} u^{-1} A_{\lambda}^{1}(u)\right| d u \\
&=M \sum_{m=0}^{\infty} \int_{\lambda\left(n_{m}\right)}^{\lambda\left(n_{m}+1\right)} u^{\gamma}\left|\frac{d}{d u} u^{-1} A_{\lambda}^{1}(u)\right| d u \\
& \leqq M \sum_{m=0}^{\infty} \lambda^{\gamma}\left(n_{m}+1\right) \int_{\lambda\left(n_{m}\right)}^{\lambda\left(n_{m}+1\right)}\left|\frac{d}{d u} u^{-1} A_{\lambda}^{1}(u)\right| d u \\
&=M \sum_{m=0}^{\infty} \lambda^{\gamma}\left(n_{m}+1\right) \int_{\lambda\left(n_{m}\right)}^{\lambda\left(n_{m}+1\right)} u^{-2}\left|B_{\lambda}^{0}(u)\right| d u \\
&=M \sum_{m=0}^{\infty} \exp \left[\lambda\left(n_{m}\right) x_{0}\right] \lambda^{\gamma-1}\left(n_{m}+1\right)\left[\lambda\left(n_{m}+1\right)-\lambda\left(n_{m}\right)\right] \\
&<\infty,
\end{aligned}
$$

by (4), since $\gamma<1$.

This completes the proof of the theorem.

REMARK. It is clear that the above series $\sum a_{n}$ is summable $|R, \lambda, k, 0|_{m}$, i.e. summable $|R, \lambda, k|_{m}$ but not summable $|A, \lambda|_{m}$. Thus for arbitrary $\left\{\lambda_{n}\right\}$, in Theorem A (i) alone does not imply (iii).

\section{REFERENCES}

1. D. Borwein, An extension of a theorem on the equivalence between absolute Rieszian and absolute Cesaro summability, Proc. Glasgow Math. Assoc. 4 (1959), 81-83.

2. K. Chandrasekhran and S. Minakshisundram, Typical means, Oxford Univ. Press, Bombay, 1952.

3. T. M. Flett, On an extension of absolute summability and some theorems of Littlewood and Paley, Proc. London Math. Soc. 7 (1957), 113-141.

4. G. H. Hardy and M. Riesz, The general theory of Dirichlet's series, Cambridge Univ. Press, Cambridge, 1915.

5. J. M. Hyslop, On the absolute summability of series by Rieszian means, Proc. Edinburgh Math. Soc. 5 (1936), 46-54.

6. S. M. Mazhar, On an extension of absolute Riesz summability, Proc. Nat. Inst. Sci. India Part A 26 (1960), 160-167.

7. J. S. Ratti, On high indices theorems, Proc. Amer. Math. Soc. 17 (1966), 10011006.

8. - Tauberian theorems for absolute summability, Proc. Amer. Math. Soc. 18 (1967), 775-781.

9. D. Waterman, On some high indices theorems, Trans Amer. Math. Soc. 69 (1950), $468-478$.

10. A. Zygmund, On certain integrals, Trans. Amer. Math. Soc. 55 (1944), 170-204.

UNIVERSITY OF SOUTH FLORIDA 